\title{
[gw22-e0252] CORRELATION BETWEEN PLATELET GELSOLIN LEVEL AND DIFFERENT TYPES OF CORONARY HEART DISEASE
}

Liu Yue, Yin Huijun, Jiang Yuerong, Xue Mei, Chen Keji Xiyuan Hospital of China Academy of Chinese Medical Sciences, Beijing, China

\subsection{6/heartjnl-2011-300867.400}

Purpose In this study, we observe the distribution of gelsolin in human platelet and plasma and its association with different types of coronary heart disease (CHD).

Methods One hundred and fourteen cases were included, with 33 stable angina pectoris (SAP) cases, 81 acute coronary syndrome (ACS) cases composed of 39 unstable angina pectoris (UAP) and 42 acute myocardial infarction (AMI) cases, and 31 healthy control participants. Gelsolin concentration in platelet rich plasma (PRP), platelet poor plasma (PPP), F-actin and Gc-globulin of PPP were determined by enzyme-linked immuno adsorbent assay (ELISA), and fluorescence intensity of CD62p and cytoplasmic calcium $\left(\mathrm{Ca}^{2+}{ }_{\mathrm{i}}\right)$ in human platelets were measured with flow cytometry.

Results Compared with the control group, the gelsolin level in PRP of UAP and AMI groups increased significantly $(\mathrm{p}<0.01)$, while gelsolin level in PPP of all the three patients group decreased markedly $(p<0.01)$, and the CD62p, $\left(\mathrm{Ca}^{2+}\right)$ $i$ of platelet, F-actin and Gc-globulin of the UAP and AMI groups increased significantly $(p<0.01)$. Compared with the SAP group, the gelsolin level in PRP, the $\left[\mathrm{Ca}^{2+}\right]_{i}$ of platelet and CD62p expression of other two groups increased significantly $(p<0.01)$, F-actin of AMI group increased markedly $(p<0.01)$, and the Gc-globulin of other two groups have no statistic deference ( $p>0.05)$.

Conclusions Platelet cytoskeleton protein dynamics vary among the different types of CHD. Gelsolin levels in PRP are markedly increased and accompanied by increasing platelet activity, platelet F-actin and $\left(\mathrm{Ca}^{2+}\right)_{\mathrm{i}}$ are closely related to ACS while gelsolin levels in PPP are markedly lower. Abnormally increased platelet gelsolin levels show high positive correlation with the level of platelet activity. Platelet gelsolin might be a new potential therapeutic target of antiplatelet therapy of ACS. 